\title{
Effect of finite beam size on the spatial and spectral response of a Fabry-Perot interferometer
}

Ibon Aramburu, Mikel Lujua, Gotzon Madariaga, María Asunción Illarramendi, Joseba Zubia

Ibon Aramburu, Mikel Lujua, Gotzon Madariaga, María Asunción Illarramendi, Joseba Zubia, "Effect of finite beam size on the spatial and spectral response of a Fabry-Perot interferometer," Proc. SPIE 10452, 14th Conference on Education and Training in Optics and Photonics: ETOP 2017, 104524E (16 August 2017); doi: 10.1117/12.2266855

SPIE Event: 14th Conference on Education and Training in Optics and Photonics, ETOP 2017, 2017, Hangzhou, China 


\title{
Effect of finite beam size on the spatial and spectral response of a Fabry-Perot interferometer
}

\author{
Ibon Aramburu*a ${ }^{\text {a }}$, Mikel Lujua ${ }^{\mathrm{b}}$, Gotzon Madariaga ${ }^{\mathrm{b}}$, María Asunción \\ Illarramendi ${ }^{\mathrm{a}}$, and Joseba Zubia ${ }^{\mathrm{c}}$ \\ a Departamento de Física Aplicada I, Escuela de Ingeniería de Bilbao, University of the Basque Country, \\ UPV/EHU, Plaza Ingeniero Torres Quevedo, 1, 48013 Bilbao, Spain \\ ${ }^{b}$ Departamento de Física de la Materia Condensada, Facultad de Ciencia y Tecnología, University of the \\ Basque Country, UPV/EHU, Apdo. 644, 48080 Bilbao, Spain \\ ${ }^{c}$ Departamento de Ingeniería de Comunicaciones, Escuela de Ingeniería de Bilbao, University of the \\ Basque Country, UPV/EHU, Plaza Ingeniero Torres Quevedo, 1, 48013 Bilbao, Spain
}

\begin{abstract}
Fabry-Perot (F-P) interferometers are commonly studied in undergraduate textbooks. Their spectral transmittance profiles are usually analyzed assuming that a plane wave is incident on the interferometer. This wave undergoes multiple reflections on the interferometer surfaces, and the interference of all these waves leads to the typical resonance structure of the spectral transmittance profile described by the Airy formula. However Fabry-Perot interferometers are commonly used in conjunction with laser beams, for example when they are used as intracavity-wavelength and longitudinal mode-selecting etalons. Although it is evident that the finite size of the beam will produce a deterioration of the filtering characteristics of the F-P interferometer, this effect is not usually analyzed in undergraduate textbooks. The aim of this work is to show students how the finite size of the incident beam influences the spatial and spectral response of the F-P interferometer. In particular it will be shown that the spectral response of the F-P interferometer can significantly differ from that predicted by the Airy formula. The theoretical approach is based on a plane-wave angular spectrum representation of the incident, transmitted, and reflected beams. The incident beam is assumed to be gaussian and the cases of normal and oblique incidence are discussed.
\end{abstract}

Keywords: Fabry-Perot, Gaussian beam, Interference, Electromagnetic optics

*Corresponding author: ibon.aramburu@ehu.es

\section{INTRODUCTION}

Beam Optics is a subject rarely discussed in undergraduate Optics textbooks [1-4]. The treatment of optical wave propagation is usually restricted to plane waves. Nevertheless they are unrealistic because they extend spatially over all of space, with constant amplitude, and carry infinite total energy. Real waves have the form of bounded beams, spatially localized, and with finite energy content. Since the

14th Conference on Education and Training in Optics and Photonics: ETOP 2017, edited by Xu Liu, Xi-Cheng Zhang, Proc. of SPIE Vol. 10452, 104524E - (c) 2017 ICO, IEEE, OSA, SPIE CCC code: $0277-786 X / 17 / \$ 18 \cdot$ doi: $10.1117 / 12.2266855$

Proc. of SPIE Vol. 10452 104524E-1 
advent of the laser the study of optical beams has experienced a strong development [5-7]. Optical beams also play an essential role in the rapidly growing research area of nano-optics [8].

In undergraduate Optics textbooks the analysis of the performance of many optical devices is usually carried out assuming that the incident wave is a plane wave. One typical example is the Fabry-Perot (FP) interferometer [1-4]. The spectral dependence of the transmittance of this device is usually deduced by adding the multiple waves generated by the reflections undergone by the incident plane wave on the surfaces of the etalon. The interference of all these waves gives rise to the characteristic spectral dependence of transmittance described by the Airy function. Nevertheless, FP interferometers are commonly used as intracavity wavelength selectors in laser cavities so that they interact not with plane waves but with laser beams. If the FP etalon is tilted an angle $\theta_{l}$ relative to the laser beam axis, the finite spatial extent of the laser beam prevents the full spatial overlap of the multiple waves generated by reflection on the surfaces of the etalon. This will produce a deterioration of the filtering characteristics of the F-P interferometer and the spectral dependence of the transmittance will be different from that predicted using plane waves.

The aim of our paper is to familiarize the student with the physics of beam optics: how optical beams are described, and how different the performance of optical devices can be when real optical beams, instead of plane waves, are used. For that purpose we have chosen the simplest type of beam provided by a laser source: the Gaussian beam [5-7], and an optical device well known by the students: the FP interferometer.

In order to simplify the analysis as much as possible we have used the simplest realization of a FP etalon: a dielectric slab of thickness $h$ and refractive index $n_{2}$ immersed in a medium of refractive index $n_{1}$. In the calculations carried out in the paper we have assumed the following values for the relevant parameters of the system: $\lambda_{H e-N e}=632.8 \mathrm{~nm}, h=4 \mathrm{~mm}, \mathrm{n}_{l}=1$, and $\mathrm{n}_{2}=2.6$ (the refractive index of $\mathrm{ZnSe}$ for $\lambda_{H e-N e}$ ).

\section{PLANE WAVES}

The transmittance and reflectance of a plane wave incident on the etalon at an angle $\theta_{l}$ to the normal can be analyzed by adding the amplitudes of the infinite number of partial waves produced by multiple reflections at the two surfaces $[1-4,9]$ :

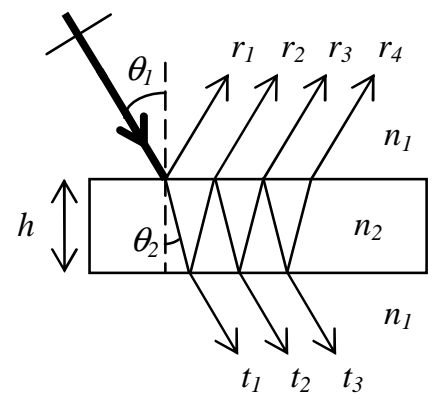

Figure 1. Reflection and transmission of a plane wave incident on the FP etalon

This derivation was first carried out by G. B. Airy in 1833 and the expressions obtained are usually known as the Airy's formulas [9]: 


$$
\begin{aligned}
& R_{F P}=\frac{F \sin ^{2}\left(\frac{\delta}{2}\right)}{1+F \sin ^{2}\left(\frac{\delta}{2}\right)} \\
& T_{F P}=\frac{1}{1+F \sin ^{2}\left(\frac{\delta}{2}\right)},
\end{aligned}
$$

where:

$$
\begin{gathered}
\delta=2 \frac{2 \pi}{\lambda_{o}} n_{2} h \cos \left(\theta_{2}\right) \\
F=\frac{4 R}{(1-R)^{2}} .
\end{gathered}
$$

In these expressions $\delta$ is the phase delay between two partial waves due to one additional round trip, $\lambda_{o}$ is the wavelength in vacuum and $R$ the reflectance of the etalon surfaces. According to the Fresnel formulae:

$$
R=\left(\frac{\bar{n}_{1}-\bar{n}_{2}}{\bar{n}_{1}+\bar{n}_{2}}\right)^{2}
$$

where:

$$
\begin{aligned}
& \bar{n}_{1}=n_{1} \cos \left(\theta_{1}\right) \\
& \bar{n}_{2}=n_{2} \cos \left(\theta_{2}\right)
\end{aligned}
$$

for TE polarization, and:

$$
\begin{aligned}
& \bar{n}_{1}=n_{1} \sec \left(\theta_{1}\right) \\
& \bar{n}_{2}=n_{2} \sec \left(\theta_{2}\right)
\end{aligned}
$$

for TM polarization.

According to equation (2), the maximum value of the etalon transmittance will be $T_{F P}^{M a x}=1$, and the minimum value $T_{F P}^{M i n}\left(\theta_{1}\right)=\frac{1}{1+F\left(\theta_{1}\right)}$. In the system we are studying the index contrast is $n_{2} / n_{l}=2.6$ and the reflectance of the etalon surfaces at normal incidence is small: $R\left(\theta_{1}=0^{\circ}\right)=0.2$. In consequence, $T_{F P}^{M i n}\left(\theta_{1}=0^{\circ}\right)=0.45$ and the filtering performance of the FP etalon at normal incidence is quite limited. However, in the case of TE polarization, when the angle of incidence increases the reflectance of the etalon surfaces also increases and the performance of the FP etalon improves (see Fig. 2.a). Finally, at 
angles of incidence high enough $\left(\theta_{l} \geq 85^{\circ}\right)$ the minimum value of the transmittance becomes practically zero $\left(T_{F P}^{M \text { in }}\left(\theta_{1}\right) \leq 0.02\right)$.

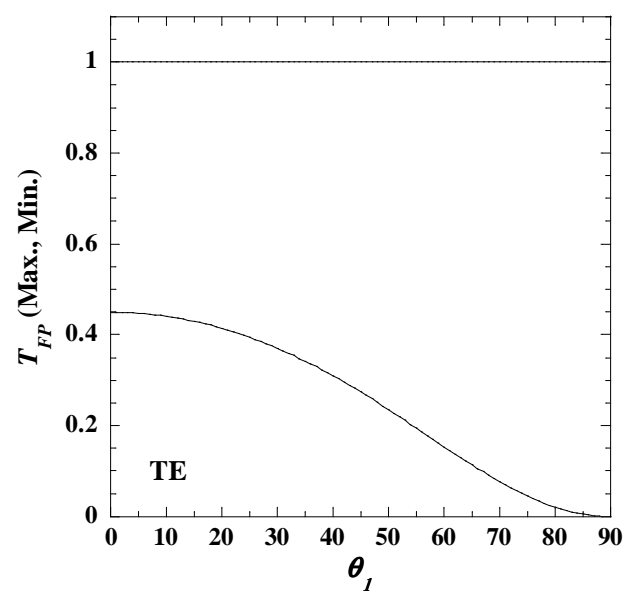

a)

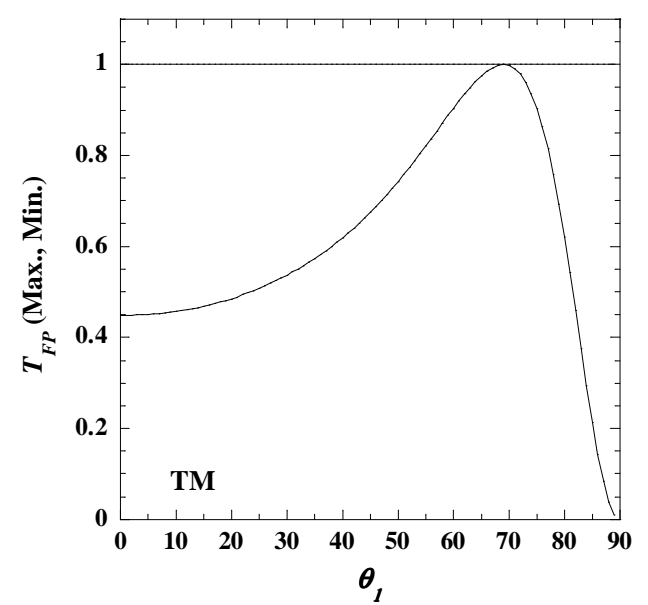

b)

Figure 2. Maximum and minimum transmittance of the FP etalon as a function of the angle of incidence (in degrees) for plane waves with: a) TE polarization; b) TM polarization

The case of TM polarization is quite different: when $\theta_{l}$ is increased from normal incidence the reflectance of the etalon surfaces decreases and becomes zero at Brewster's angle $\left(\theta_{B}=69^{\circ}\right)$. The incident wave is totally transmitted by the FP etalon at this angle $\left(T_{F P}^{M i n}\left(\theta_{B}\right)=T_{F P}^{M a x}\left(\theta_{B}\right)=1\right)$ independently of the value of the wavelength. Therefore a good filtering performance of the FP etalon for TM polarization can only be achieved at very high angles of incidence (see Fig. 2.b).

The transmission of the FP etalon is maximum $\left(T_{F P}^{M a x}=1\right)$ when

$$
\delta=2 \frac{2 \pi}{\lambda_{o}} n_{2} h \cos \left(\theta_{2}\right)=2 m \pi, \quad \mathrm{m}=\text { any integer }
$$

By using $\lambda_{o}=c / v$, where $c$ is the velocity of light in vacuum and $v$ is the optical frequency, condition (10) can be written as

$$
v_{m}=m \frac{c}{2 n_{2} h \cos \left(\theta_{2}\right)}
$$

Thus, for a fixed $\theta_{1}$, the frequencies of unity transmission are equally spaced. The separation between two consecutive resonance frequencies is called "free spectral range":

$$
\Delta v \equiv v_{m+1}-v_{m}=\frac{c}{2 n_{2} h \cos \left(\theta_{2}\right)} .
$$


The spectral dependence of the etalon transmittance for several angles of incidence is shown in Fig. 3.a (TE polarization) and Fig. 3.b (TM polarization). As the free spectral range depends on $\theta_{l}$, the frequencies have been normalized by $\Delta v$ in order to make them comparable.

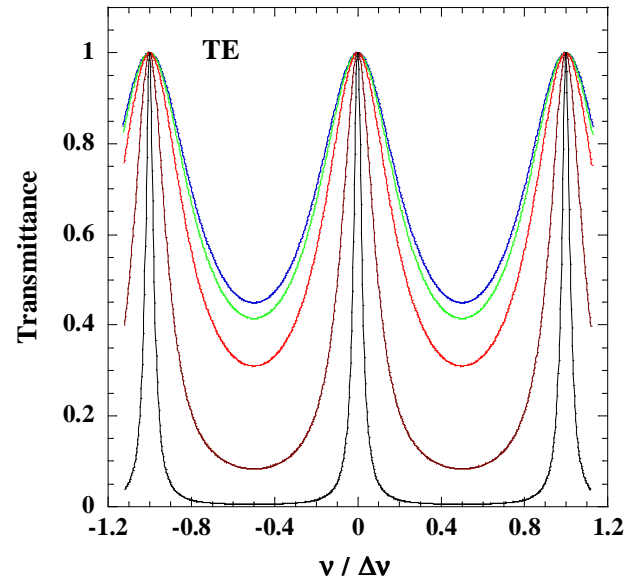

a)

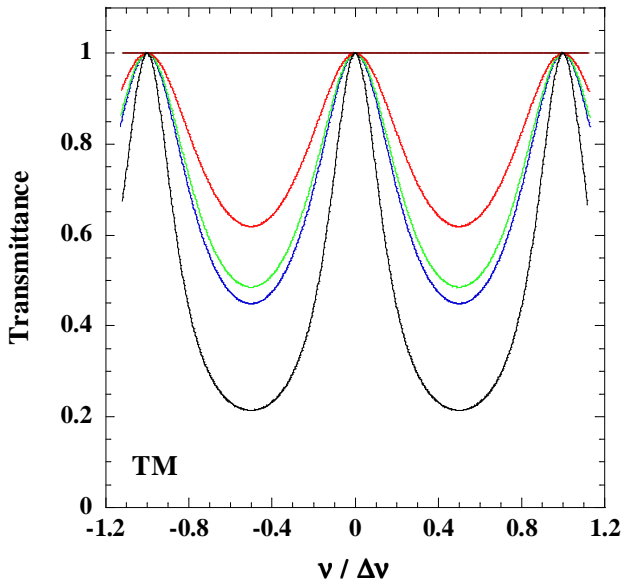

b)

Figure 3. Spectral dependence of the etalon transmittance for several angles of incidence $\left(\theta_{l}=0^{\circ}\right.$ : blue; $\theta_{l}=20^{\circ}$ : green; $\theta_{l}=40^{\circ}$ : red; $\theta_{l}=69^{\circ}$ : brown; $\theta_{l}=85^{\circ}$ : black). The incident waves are plane waves with: a) $\mathrm{TE}$ polarization; b) TM polarization

In the case of TE polarization it can be observed that when $\theta_{l}$ increases not only the contrast between the maximum and minimum transmittance increases but also the peaks become sharper.

The use of the FP etalon as a spectrum analyzer requires that the width of the transmission peaks be small and the free spectral range be wide. Thus, the resolution of the FP etalon is usually characterized by the "finesse" defined as the ratio of the free spectral range to the full width of the transmission peaks at their half-maximum values (FWHM):

$$
\text { Finesse } \equiv \frac{\Delta v}{\text { FWHM }}
$$

The dependence of the finesse of the FP etalon on the angle of incidence for TE and TM polarization is shown in Fig. 4. In the case of TM polarization (Fig. 4.b) the finesse is not defined for $24^{\circ} \leq \theta_{1} \leq 81^{\circ}$, because in that range $T_{F P}^{\text {Min }}\left(\theta_{1}\right)>\frac{T_{F P}^{M a x}\left(\theta_{1}\right)}{2}$. 


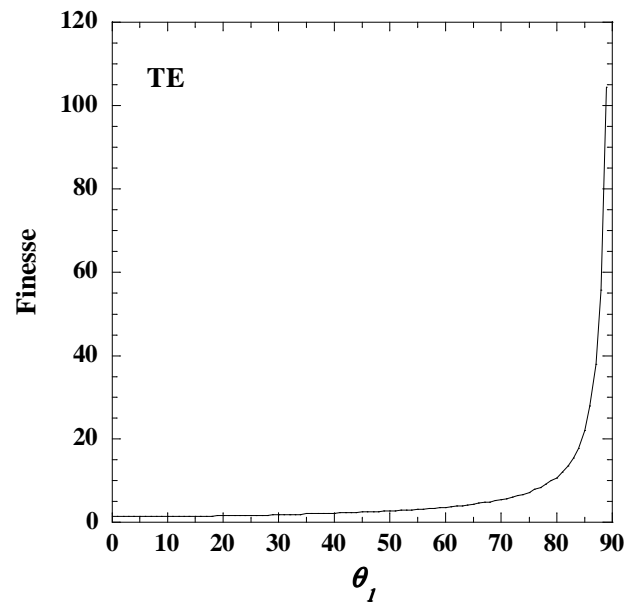

a)

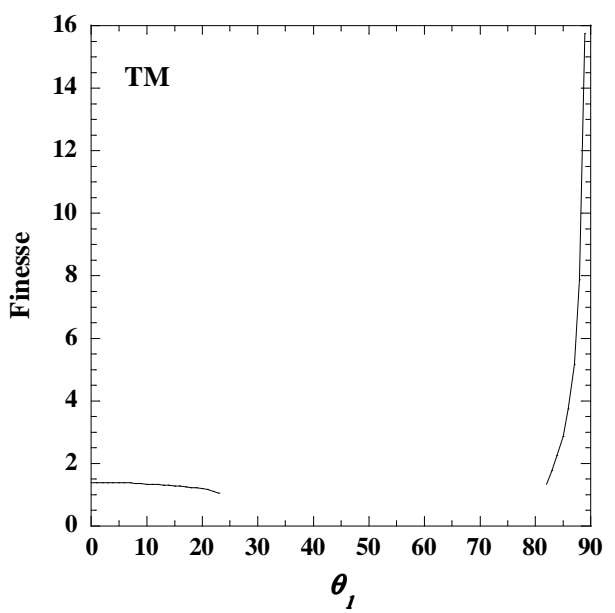

b)

Figure 4. Finesse of the FP etalon as a function of the angle of incidence (in degrees) for plane waves with: a) TE polarization; b) TM polarization

\section{GAUSSIAN BEAMS}

\subsection{Main Features}

Gaussian beams are the simplest type of beam provided by a laser source [3-7, 10]. The spatial structure of the fundamental mode $\mathrm{TEM}_{00}$ propagating along the $\mathrm{Z}$ axis is given by:

$$
\frac{E(x, y, z)}{E_{o}}=\frac{w_{o}}{w(z)} \exp \left[-\frac{x^{2}+y^{2}}{w^{2}(z)}\right] \exp \left[-i k\left(z+\frac{x^{2}+y^{2}}{2 R(z)}\right)\right] \exp \left[i \tan ^{-1}\left(\frac{z}{z_{R}}\right)\right]
$$

where $k=\frac{2 \pi}{\lambda}$ is the wavenumber of light in the medium, and

$$
\begin{aligned}
& w(z)=w_{o} \sqrt{1+\left(\frac{z}{z_{R}}\right)^{2}} \\
& R(z)=z\left[1+\left(\frac{z_{R}}{z}\right)^{2}\right] \\
& z_{R}=\frac{\pi w_{o}^{2}}{\lambda} .
\end{aligned}
$$

According to eq. (14) at any transverse plane $z=$ constant the beam intensity decreases by a factor $1 / \mathrm{e}^{2}$ at the radial distance $\sqrt{x^{2}+y^{2}}=w(z)$. As most of the power carried by the beam $(86 \%)$ is within a circle 
of radius $w(z)$ in the transverse plane, $w(z)$ is usually regarded as the beam radius (or beam width). The beam radius is minimum $\left(w_{o}\right)$ at the plane $\mathrm{z}=0$ (beam waist) and increases monotonically with $\mathrm{z}$ (see eq. (15)). The minimum value of the beam radius $w_{o}$ is thus known as the "waist radius", and $2 w_{o}$ is usually called the "spot size". At a distance $z_{R}$ from the waist (Rayleigh range) the cross-sectional area of the beam doubles (see eq. (15)). Therefore the distance $2 z_{R}$ can be considered as an estimation of the beam waist dimension along the $\mathrm{Z}$ axis and is called "depth of focus". At large distances from the waist ( $z \gg z_{R}$ ) the beam diverges as a cone of full-angular width:

$$
\Theta=\frac{2 \lambda}{\pi \mathrm{w}_{o}}
$$

The smaller the waist radius $w_{o}$, the smaller the depth of focus and the faster the beam diverges. According to eqs. (14)-(18), once fixed the values of $E_{o}$ and $\lambda$, the shape of the Gaussian beam is governed only by the waist radius $w_{o}$.

\subsection{Transmission of a Gaussian beam through a Fabry-Perot etalon}

In order to analyze the transmission of a Gaussian beam through the FP etalon considered in section 2 we have decomposed the Gaussian beam into plane waves that propagate in all three dimensions (angular spectrum representation) [8]. For each incident plane wave we have calculated the reflected and transmitted plane waves using the standard procedure outlined in section 2 but generalized to include three-dimensional wavevectors. Finally the total field on a plane $\Pi_{t}\left(\Pi_{\mathrm{r}}\right)$ at a distance $\mathrm{z}_{\mathrm{t}}\left(\mathrm{z}_{\mathrm{r}}\right)$ from the etalon (see Fig. 5) has been calculated by adding all transmitted (reflected) complex plane waves with their appropriate phases.

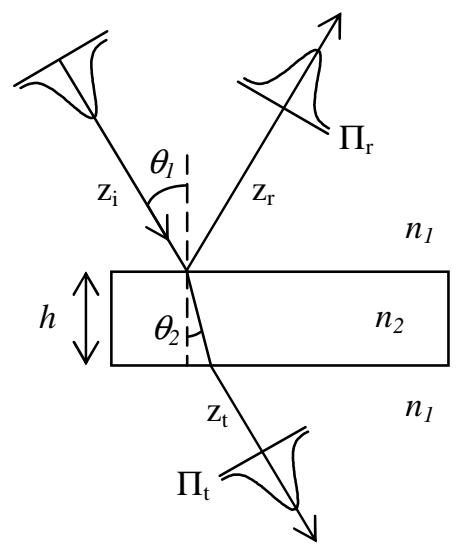

Figure 5. Reflection and transmission of a Gaussian beam incident on the FP etalon

The transmittance of the FP etalon has been calculated as the ratio of the transmitted energy flux (obtained by spatial integration on the $\Pi_{t}$ plane of the intensity of the transmitted beam) to the incident energy flux. The same procedure has been followed to obtain the reflectance of the FP etalon. The number of points in k-space has been conveniently chosen in order to get good convergence.

According to section 3.1, if the wavelength $\lambda$ and the peak amplitude of the field $E_{o}$ are known, the only parameters required to completely characterize the incident Gaussian beam are the direction of the beam axis, the location of the waist, and the waist radius $w_{0}$. In our simulations the direction of the beam axis is 
specified by the angle of incidence $\theta_{1}$. The waist is assumed to be located at $\mathrm{z}_{\mathrm{i}}=10 \mathrm{~cm}$. from the etalon and we have analyzed the transmission of the FP etalon for three different values of the waist radius: $\mathrm{w}_{\mathrm{o}}=0.25 \mathrm{~mm}$., $0.5 \mathrm{~mm}$., and $1 \mathrm{~mm}$. The planes $\Pi_{\mathrm{t}}$ and $\Pi_{\mathrm{r}}$ are assumed to be at a distance $\mathrm{z}_{\mathrm{t}}=\mathrm{z}_{\mathrm{r}}=10 \mathrm{~cm}$. from the etalon. The values of $T_{F P}$ and $R_{F P}$ have been calculated for a large number of wavelengths close to $\lambda_{\mathrm{He}-\mathrm{Ne}}=632.8 \mathrm{~nm}$ in order to obtain the spectral response of the FP etalon.

The results obtained in the calculations show that the maximum transmittance is achieved for the same wavelengths and angles of incidence as in the plane-wave case (see Fig. 6), so that eqs. (11)-(12) are still valid for Gaussian beams.

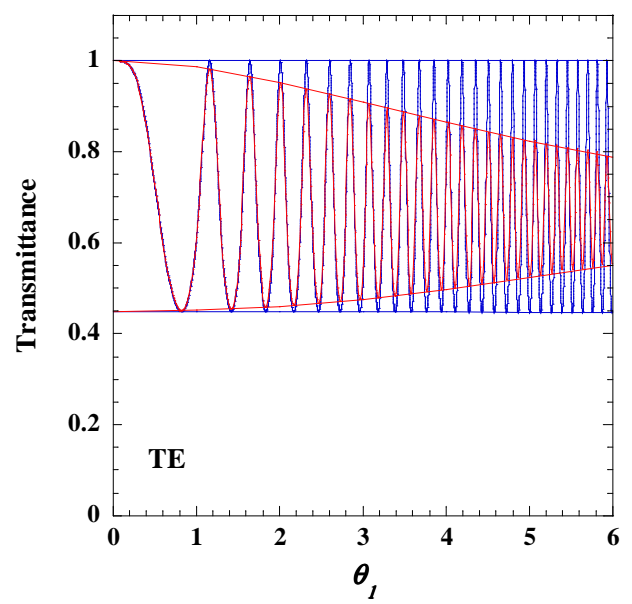

Figure 6. Transmittance of the FP etalon as a function of the angle of incidence (in degrees) for plane waves (blue line) and Gaussian beams ( $\mathrm{w}_{\mathrm{o}}=0.25 \mathrm{~mm}$., red line) with TE polarization and wavelength $\lambda=632.815 \mathrm{~nm}$.

However the maximum and minimum values of the etalon transmittance significantly differ from those obtained with plane waves (see Fig. 7). When the angle of incidence increases from normal the filtering performance of the FP etalon worsens. The smaller the waist radius $w_{o}$ of the incident Gaussian beam, the faster the deterioration of the filtering efficiency. Thus, if the waist radius $w_{o}$ is small enough, for a certain angle of incidence the FP etalon stops filtering. Surprisingly, for high values of $\theta_{l}$ the filtering ability of the FP etalon is recovered. 

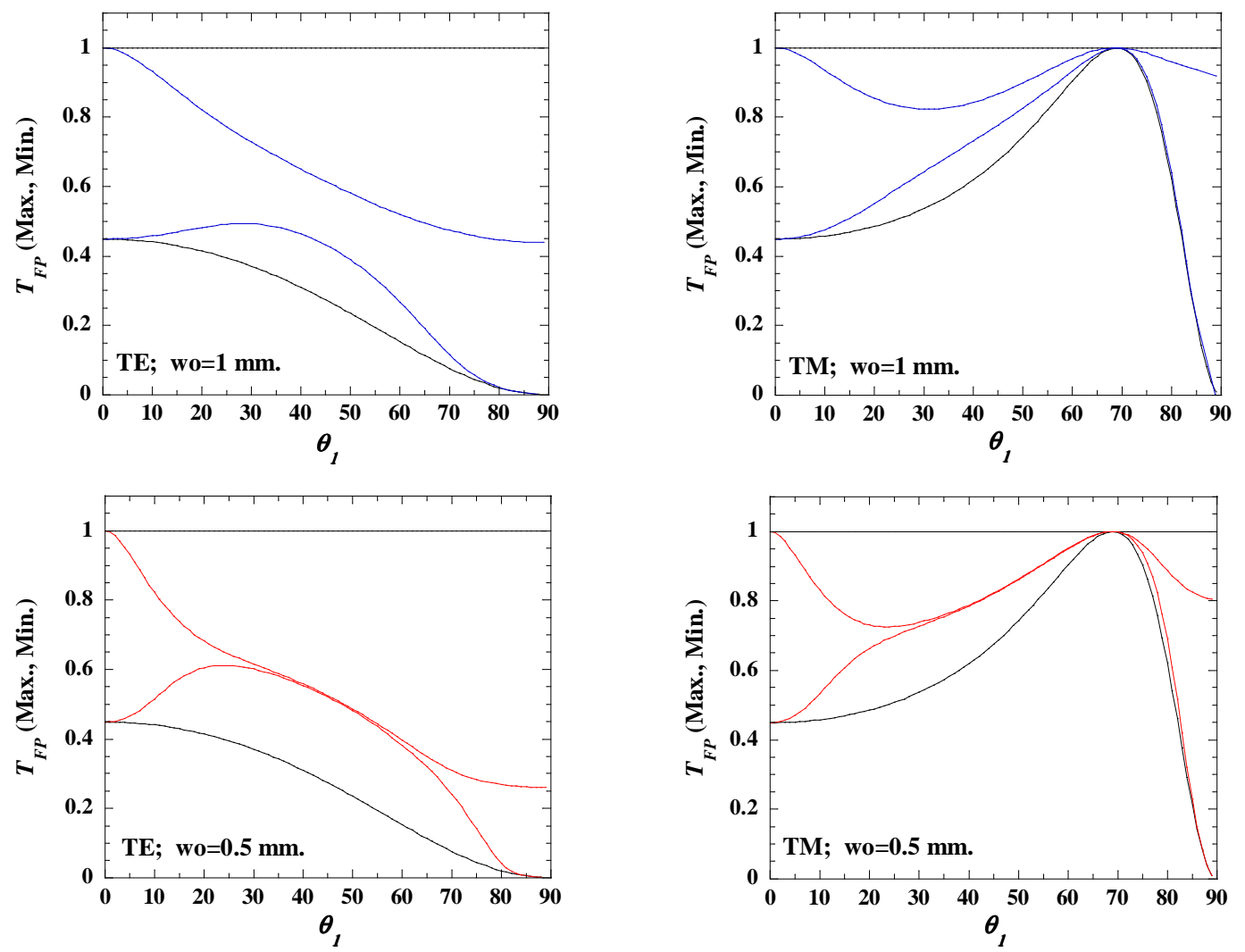

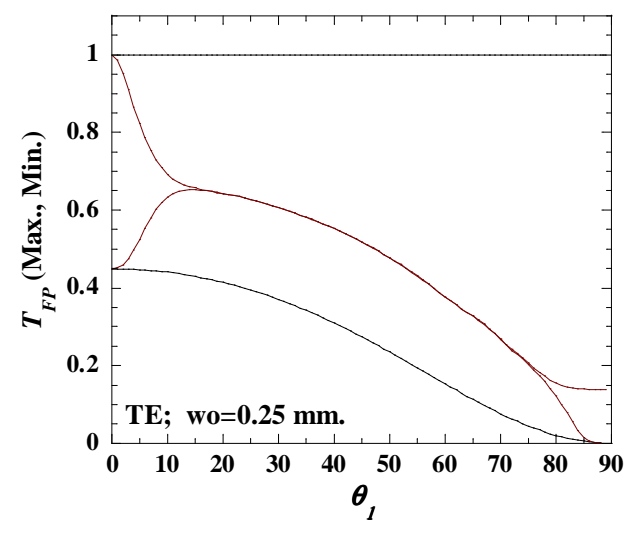

a)

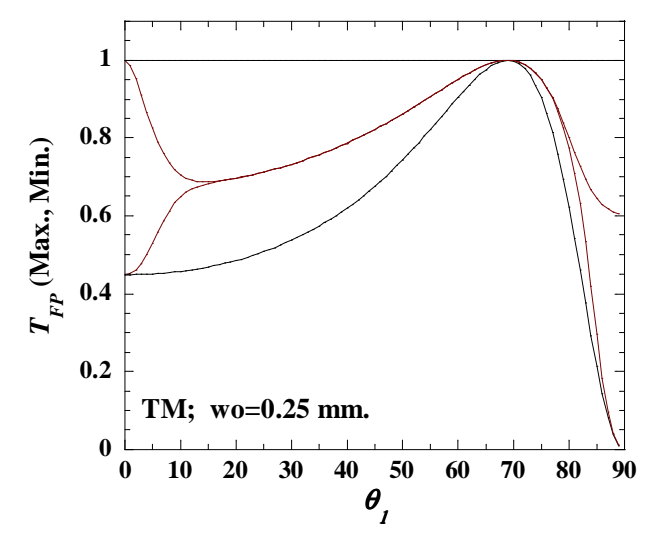

b)

Figure 7. Maximum and minimum transmittance of the FP etalon as a function of the angle of incidence (in degrees) for plane waves (black) and Gaussian beams of different waist radius (color) with: a) TE polarization; b) TM polarization

The spectral response of the etalon for several angles of incidence is represented in Fig. 8. A comparison with the results obtained with plane waves (Fig. 3) shows the strong degradation of the filtering efficiency of the etalon when is illuminated by a Gaussian beam, especially for TE polarization (compare Fig 3.a with Fig. 8.a). 


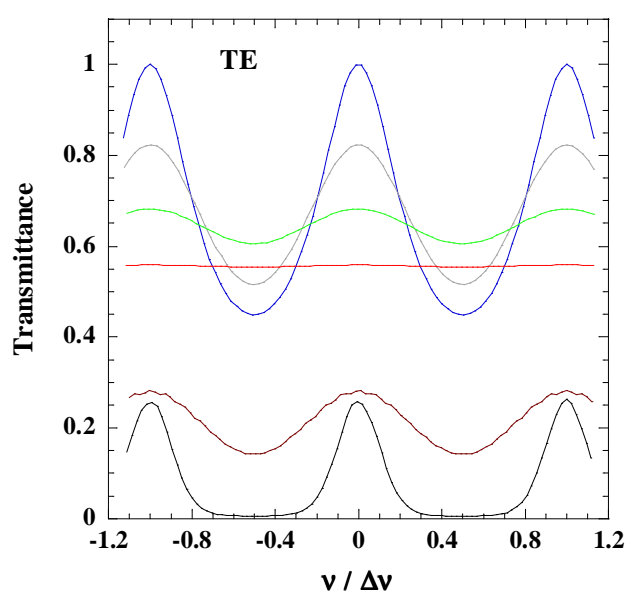

a)

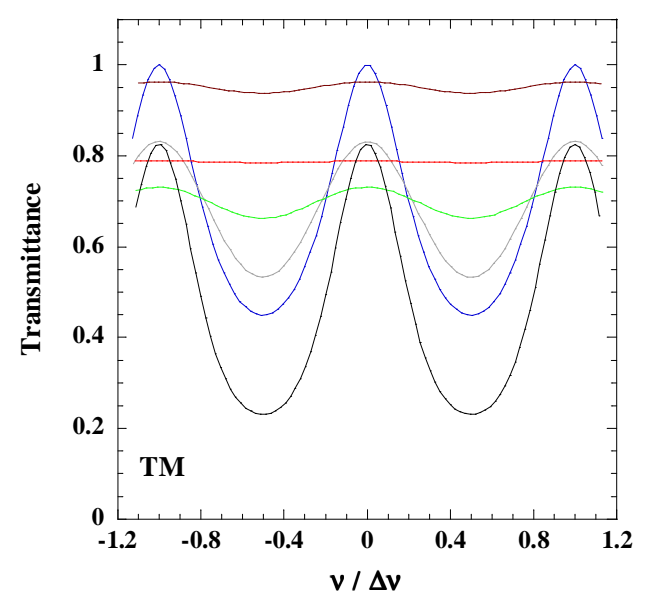

b)

Figure 8. Spectral dependence of the etalon transmittance for several angles of incidence $\left(\theta_{l}=0^{\circ}\right.$ : blue; $\theta_{l}=10^{\circ}$ : grey; $\theta_{l}=20^{\circ}$ : green; $\theta_{l}=40^{\circ}$ : red; $\theta_{l}=75^{\circ}$ : brown; $\theta_{l}=85^{\circ}$ : black). The incident wave is a Gaussian beam of waist radius $\mathrm{w}_{\mathrm{o}}=0.5 \mathrm{~mm}$. with: a) TE polarization; b) TM polarization.

The dependence of the finesse of the FP etalon (calculated according to eq. (13)) on the angle of incidence for TE and TM polarization is shown in Fig. 9.

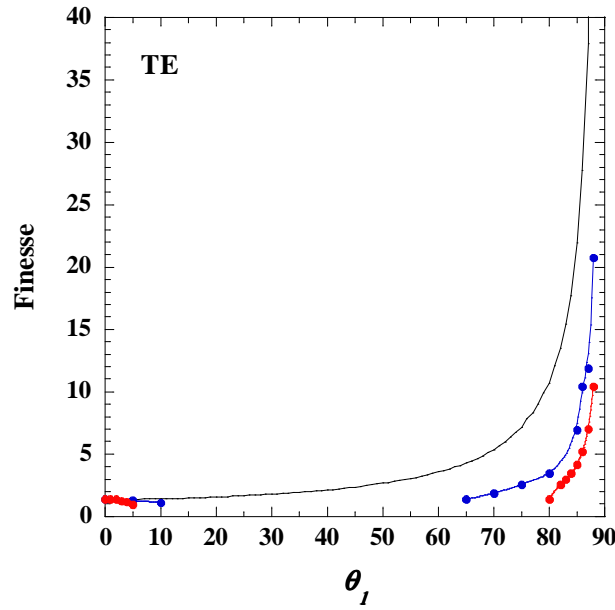

a)

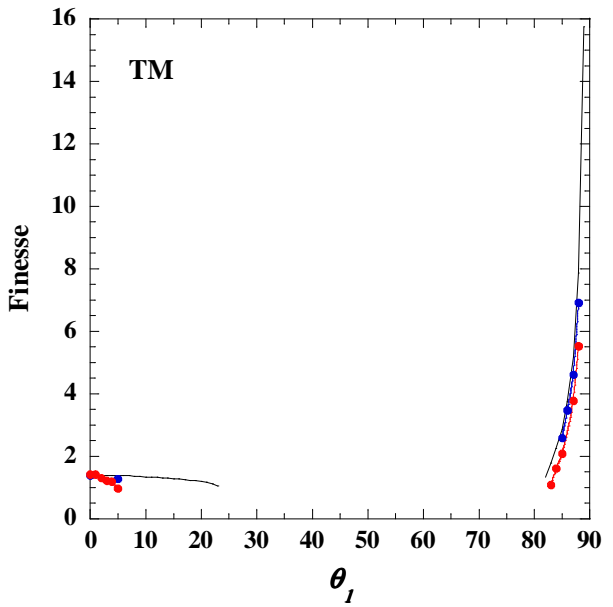

b)

Figure 9. Finesse of the FP etalon as a function of the angle of incidence (in degrees) for plane waves (black lines) and Gaussian beams of different waist radius ( $\mathrm{w}_{\mathrm{o}}=1 \mathrm{~mm}$. (blue points); $\mathrm{w}_{0}=0.5 \mathrm{~mm}$. (red points)) with: a) TE polarization; b) TM polarization

It can be observed that for high angles of incidence the finesse is greater for TE polarization than for TM polarization. Therefore, on the basis of the results obtained for plane waves, it is expected that the filtering performance of the FP etalon be better for TE polarization than for TM. However this is not so clear because, for example, the contrast between the maximum and minimum transmittance ( 
$\left.T_{F P}^{M a x}\left(\theta_{1}\right)-T_{F P}^{M i n}\left(\theta_{1}\right)\right)$ is greater for TM polarization (see Figs. 7, 8). Therefore, when the maximum transmittance is less than 1 (as usually happens when the FP etalon is illuminated by a Gaussian beam), the value of the finesse is not enough to characterize the filtering performance of the etalon.

\section{DISCUSSION}

The filtering of the FP etalon is the result of the interference of the multiple waves generated by reflection at the two surfaces of the etalon. That requires the overlapping of the waves. In the case of an incident plane wave its infinite spatial extent assures the complete overlapping of the multiple waves for any angle of incidence, giving rise to the typical resonance structure of the spectral transmittance profile described by the Airy's formula.

However, in the case of an incident Gaussian beam, the finite transverse spatial extent of the beam prevents the complete overlapping of the waves except at normal incidence.

Let us considered, for example, a small portion of a perfectly collimated beam of finite transverse extent "A" incident on the etalon (red line in Fig. 10). The fraction that emerges from the etalon after 2 reflections is shifted a distance:

$$
\Delta x=2 \mathrm{x}=2\left(h \tan \theta_{2}\right)=h \frac{2\left(n_{1} / n_{2}\right) \sin \theta_{1}}{\sqrt{1-\left(n_{1} / n_{2}\right)^{2} \sin ^{2} \theta_{1}}}
$$

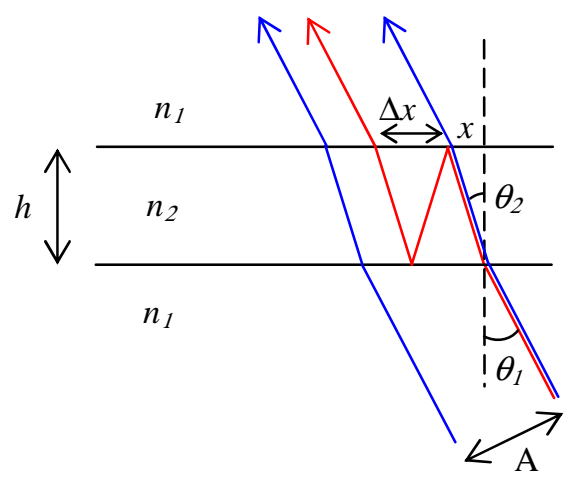

Figure 10. Transmission of a Gaussian beam incident on the FP etalon at low angle of incidence

As the angle of incidence $\theta_{l}$ increases from normal, $\Delta x$ increases, the overlapping of the partial waves decreases and the filtering efficiency of the FP etalon worsens. This result explains the performance of the FP etalon at low angles of incidence obtained in section 3 (see Fig. 7). Nevertheless $\Delta x$ is a strictly increasing function of $\theta_{1}$, so it cannot explain why the filtering ability of the FP etalon is recovered at high angles of incidence. 
The reason is that the relevant magnitude to characterize the overlapping of the waves is not $\Delta x$ but $\Delta s$, the relative displacement between two consecutive waves in the direction of the transmitted beam (see Fig. 11):

$$
\Delta s=\Delta x \cos \theta_{1}=h \frac{\left(n_{1} / n_{2}\right) \sin \left(2 \theta_{1}\right)}{\sqrt{1-\left(n_{1} / n_{2}\right)^{2} \sin ^{2} \theta_{1}}}
$$

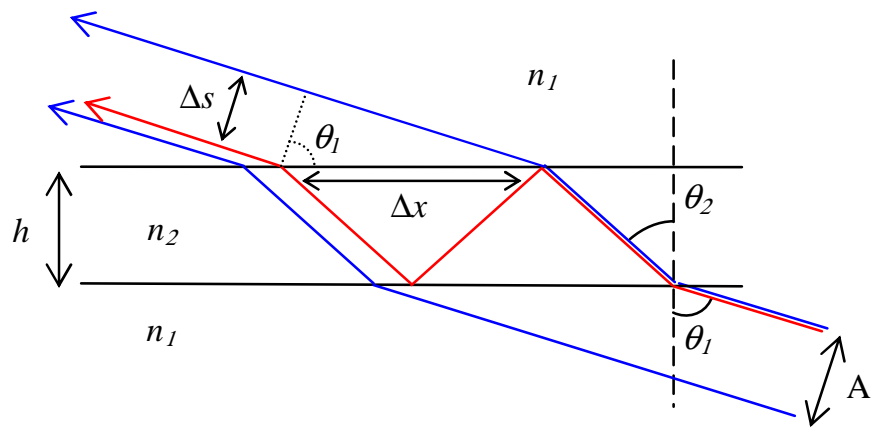

Figure 11. Transmission of a Gaussian beam incident on the FP etalon at high angle of incidence

When $\theta_{l}$ is increased the distance within the etalon between two consecutive waves $(\Delta x)$ increases. However, that distance decreases (by a factor $\cos \theta_{l}$ ) when the waves leave the etalon (see Fig. 11).

The dependence of $\Delta s$ on the angle of incidence for the FP etalon used in our study $\left(n_{1}=1, n_{2}=2.6\right.$; $\mathrm{h}=4 \mathrm{~mm}$.) is shown in Fig. 12.

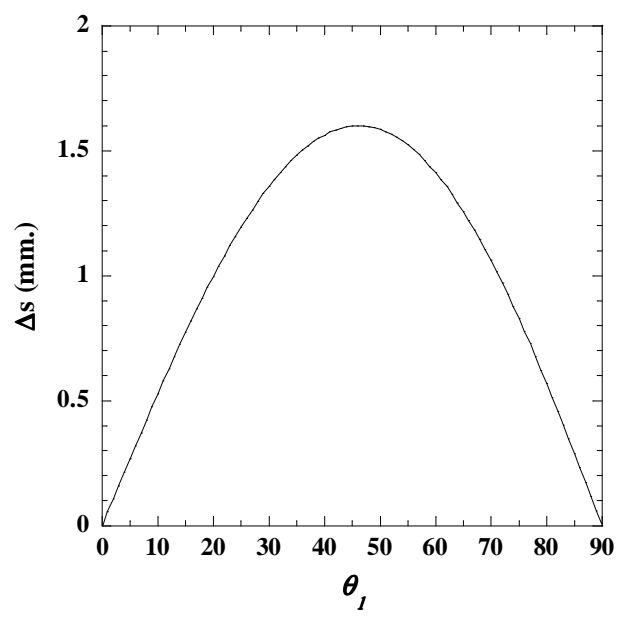

Figure 12. Displacement $\Delta s$ (in $\mathrm{mm}$.) as a function of the angle of incidence (in degrees) for the FP etalon used in our study

It can be observed that $\Delta s$ is maximum for $\theta_{1} \cong 45^{\circ}$. Thus it is expected that the greatest overlap of the waves, and therefore the best filtering efficiency of the FP etalon, occurs at both low and high angles of 
incidence. When the relative displacement between two consecutive waves $(\Delta s)$ is greater than the spot size $\left(2 w_{o}\right)$ of the incident Gaussian beam:

$$
\Delta s\left(\theta_{1}\right) \geq 2 w_{o}
$$

the partial beams generated by reflection at the surfaces of the etalon do not overlap and the FP etalon stops filtering. Eq. (21) and Fig. 12 can be used to deduce the interval of values of $\theta_{1}$ for which the etalon will not filter. For example, for the values of $w_{o}$ used in section 3:

$w_{o}=1 \mathrm{~mm} .: \quad$ Filtering is achieved for all $\theta_{l}$,

$w_{o}=0.5 \mathrm{~mm} .: \quad 21^{\circ} \leq \theta_{1} \leq 72^{\circ}$,

$w_{o}=0.25 \mathrm{~mm} .: \quad 10^{\circ} \leq \theta_{1} \leq 81^{\circ}$,

in good agreement with the intervals obtained in the simulations (see Fig. 7).

In these intervals the transmittance of the FP etalon does not depend on the waist radius of the Gaussian beam (see Fig. 13). The partial beams generated by reflection at the surfaces of the etalon do not overlap and the transmittance of the etalon can be calculated by adding the intensities of the multiple beams transmitted:

$$
T_{F P}=T^{2}\left(1+R^{2}+R^{4}+\ldots\right)=\frac{T^{2}}{1-R^{2}}=\frac{(1-\mathrm{R})^{2}}{1-R^{2}}
$$

where $T$ and $R$ are the transmittance and the reflectance of the etalon surfaces, respectively. The simulation of the curve $T_{F P}$ obtained using eqs. (22) and (5) confirms this result (green line in Fig. 13).

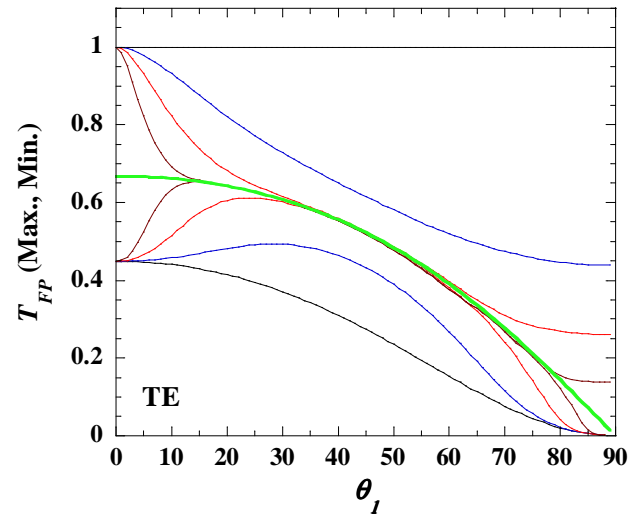

a)

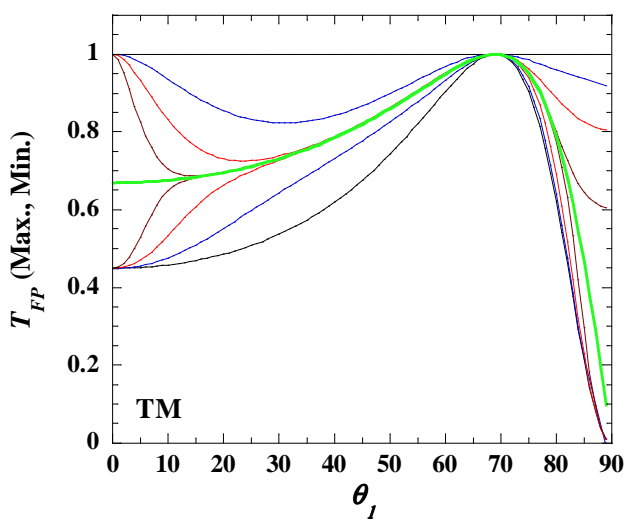

b)

Figure 13. Maximum and minimum transmittance of the FP etalon as a function of the angle of incidence (in degrees) for plane waves (black) and Gaussian beams of different waist radius $\left(\mathrm{w}_{\mathrm{o}}=1 \mathrm{~mm}\right.$.: blue; $\mathrm{w}_{\mathrm{o}}=0.5 \mathrm{~mm}$.: red; $\mathrm{w}_{\mathrm{o}}=0.25 \mathrm{~mm}$.: brown) with: a) TE polarization; b) TM polarization. The green line represents the transmittance obtained by adding the intensities of the multiple beams transmitted (no interference among the beams) (eq. (22)). 


\section{CONCLUSIONS}

The analysis of the transmittance of a FP etalon when is illuminated by a Gaussian beam is a good way of introducing the physics of optical beams to students. On the one hand, the performance of the FP etalon when is illuminated by plane waves is quite well known by students. On the other hand students can realize how essential properties of optical beams, as their finite tranverse spatial extent, can strongly modify the performance of optical devices, that can be quite different from that predicted using plane waves.

\section{ACKNOWLEDGEMENTS}

This work has been funded by the Fondo Europeo de Desarrollo Regional (FEDER); by the Ministerio de Economía y Competitividad under project TEC2015-638263-C03-01-R; by the GobiernoVasco/Eusko Jaurlaritza under projects IT933-16-13, IT-779-13 and ELKARTEK (KK-2016/0030 and KK2016/0059); and by the University of the Basque Country (UPV/EHU) under program UFI11/16.

\section{REFERENCES}

[1] E. Hetch, "Optics", 5 ${ }^{\text {th }}$ edition (Global Edition), Pearson Education Limited, 2017.

[2] F.L. Pedrotti, and L.S. Pedrotti, "Introduction to Optics", $2^{\text {nd }}$ edition, Prentice-Hall, 1993.

[3] B.E.A. Saleh, and M.C. Teich, "Fundamentals of Photonics", $2^{\text {nd }}$ edition, John Wiley and Sons: New York, 2007.

[4] R. Guenther, "Modern Optics”, John Wiley and Sons: New York, 1990.

[5] A.E. Siegman, "Lasers", University Science Books, 1986.

[6] O. Svelto, "Principles of Lasers", $4^{\text {th }}$ edition, Springer, 1998.

[7] W.T. Silfvast, "Laser Fundamentals", $2^{\text {nd }}$ edition, Cambridge University Press, 2004.

[8] L. Novotny, and B. Hecht, "Principles of Nano-Optics", $2^{\text {nd }}$ edition, Cambridge University Press, 2012.

[9] M. Born and E. Wolf, "Principles of Optics", $7^{\text {th }}$ edition, Cambridge University Press, 2005.

[10] Michael Bass (Ed.), "Handbook of Optics" (sponsored by the OSA), Vol. I, $2^{\text {nd }}$ edition, MacGrawHill, 1995. 